\title{
CD34 E CASPASE-3: COMPARAÇÃO, CORRELAÇÃO DE EXPRESSÃO E QUANTIFICAÇÃO IMUNOISTOQUÍMICA NO ADENOCARCINOMA GÁSTRICO
}

\section{CD34 and caspase-3: expression, quantification comparison and correlation in gastric adenocarcinoma}

\author{
Paulo Henrique Freitas Farias SILVA, Ronaldo Mafia CUENCA, Jurandir Marcondes RIBAS-FILHO, \\ Carmen Australia Paredes Marcondes RIBAS, Osvaldo MALAFAIA, \\ Samuel DOBROWOLSKI, Fabio Roberto BORA
}

\begin{abstract}
Silva PHFF, Cuenca RM, Ribas-Filho JM, Ribas CAPM, Malafaia O, Dobrowolski S, Bora FR. CD34 e Caspase-3: comparação, correlação de expressão e quantificação imunoistoquímica no adenocarcinoma gástrico. ABCD Arq Bras Cir Dig 2008;21(4): 180-4

RESUMO - Racional - O câncer gástrico continua a desafiar a medicina moderna a compreender o seu comportamento biológico e a melhorar seus índices de cura cirúrgica e taxa de sobrevivência. Objetivo - Comparar e correlacionar a expressão e a quantificação dos marcadores caspase-3 e CD34 no adenocarcinoma gástrico com fatores clínico-patológicos e com os tumores gástricos proximais e distais. Método - Foi utilizada análise imunoistoquímica quantitativa com os anticorpos anti-caspase-3 policlonal de coelho e CD43 monoclonal de rato, para marcar células do adenocarcinoma gástrico em 26 casos com CD34 e 22 com caspase-3 oriundos de blocos parafinados. As lâminas foram lidas para os parâmetros índice de marcagem em porcentagem, e densidade óptica em pixels. A leitura foi realizada pelo sistema SAMBA de citofotometria de imagem. Resultados - As análises mostraram que a expressão dos marcadores caspase-3 e CD34 são elevadas, onde a média e o desvio-padrão para o índice de marcagem foram, respectivamente $87,72 \% \pm 7,89$ e $84,86 \% \pm 9,17$ e, para a densidade óptica, foram 60,75 $\pm 7,46$ e 49,84 \pm 8.48 . Quando compararam-se os marcadores com a densidade óptica encontrou-se diferenças significativas entre a densidade óptica de caspase-3 $(60,94)$ e a do CD34 (50,27) com $P<0.001$. A correlação entre os marcadores foi positiva para a densidade óptica de CD34 versus o índice de marcagem de $\mathrm{CD} 34$ com $P=0.006$, $\mathrm{r}=0,520$ e para densidade óptica de caspase- 3 versus índice de marcagem de caspase- 3 , com $P=0.015, \mathrm{r}=0.512$. Não encontrou-se correlação dos marcadores quando compararam-se os índices de marcagem e as densidades ópticas com a curvatura gástrica, diferenciação tumoral, classificação de Borrmann, padrão tumoral ou invasão linfática. Os parâmetros clínicos sexo e idade também não se correlacionaram com os marcadores. A correlação com o local do tumor mostrou diferença entre os tumores de localização distal e proximal, onde a média da densidade de CD34 nos distais $(52,44 \pm 7,72)$ é maior do que a dos proximais $(45,67 \pm 6,06)$ com $P=0.023$. Conclusão - As expressões dos índices de caspase-3 e CD34 são elevados para o adenocarcinoma gástrico A comparação da densidade óptica de caspase-3 é maior do que a do CD34. Há correlação linear positiva entre a densidade óptica e o índice de marcagem tanto para CD34 quanto para caspase-3. Não há correlação da expressão e quantificação entre os marcadores caspase-3 e CD34 e os fatores clínico-patológicos estudados. A densidade óptica de CD34 no adenocarcinoma gástrico distal é maior do que a densidade óptica no proximal sugerindo que apresentam diferenças biológicas relacionadas com a localização do tumor.

DESCRITORES - CD34. Caspase-3. Citofotometria.
\end{abstract}

\section{INTRODUÇAO}

O câncer gástrico continua a desafiar a medicina moderna em compreender seu comportamento biológico e a melhorar os índices de cura e de sobrevivência. O adenocarcinoma gástrico é o tipo histológico mais frequente (95\%) e apresenta várias anomalias cromossômicas e genéticas. A análise citológica revela que tumores gástricos com grande proporção de células aneuplóides e com quantidade acima do normal de DNA nuclear, estão associados com mau prognóstico nos estágios precoce e avançados. Com

Trabalho realizado na Faculdade de Medicina do Planalto Central em Brasília, DF, Brasil.

Correspondência: Paulo Henrique Freitas Farias Silva, e-mail: ipem@evangelico.org.br o desenvolvimento da imunoistoquímica foi possível investigar novos métodos de estudo das alterações nucleares e citoplasmáticas.

Novas técnicas de imunoistoquímica têm criado soluções como a categorização dos tumores indiferenciados, determinação do sítio de origem de metástases tumoral, e subclassificação de tumores em vários órgãos com maior exatidão histopatológica. A análise pelo sistema informatizado SAMBA 4000 vem contribuir para diminuir o grau de subjetividade dos métodos empregados .

Este estudo tem por objetivos: 1. determinar e comparar a expressão e a quantificação dos imunomarcadores CD34 e caspase-3 no adenocarcinoma gástrico; 2 . correlacionar a expressão e a quantificação deles com fatores clínicos e patológicos; 3 . comparar e quantificar suas expressões nos adenocarcinomas gástricos proximais e distais. 


\section{MÉTODOS}

Este estudo foi realizado na Faculdade de Medicina do Planalto Central em Brasília e no Instituto de Pesquisas Médicas do Hospital Universitário Evangélico de Curitiba/ Faculdade Evangélica do Paraná, em Curitba. Foi aprovado pelo Comitê de Ética em Pesquisa da Sociedade Evangélica Beneficente de Curitiba. É retrospectivo com delineamento tipo transversal não controlado.

O material inicial consistiu de casos de adenocarcinoma gástrico fixados em formol e conservados em blocos de parafina oriundos de pacientes gastrectomizados do Hospital Regional do Gama no Distrito Federal, Hospital e Maternidade Dom Orione (centro de alta complexidade oncológica de Araguaína) e no Laboratório de Histopatologia e Citologia de Lins, em Penápolis, SP e CITOLAB Laboratório de Citopatologia em Curitiba.

Foi realizada a seleção de lâminas que melhor representassem o diagnóstico da massa tumoral ressecada no procedimento cirúrgico, e o bloco de parafina correspondente separado para análise. Foram coletados 60 blocos de 1998 a 2006 submetidos à coloração pela Hematoxilina-Eosina (HE) para confirmação do diagnóstico inicial. As lâminas em HE que apresentaram defeitos de técnica, diagnósticos divergentes ou material insuficiente causaram exclusão de 10 blocos, restando assim 50, que foram submetidos a cortes de 3 micra. As lâminas passaram pelo processo técnico de imunoistoquímica e leitura inicial da marcagem celular do anti-CD34 e anti-caspase-3. As que apresentaram marcagem divergentes do controle positivo foram excluídas assim como o bloco parafinado correspondente.

Os marcadores utilizados foram anti-caspase- 3 humana (anti-CPP32) anticorpo policlonal de coelho na concentração $1 \mathrm{mg} / \mathrm{mL}$ código $n^{\circ} \mathrm{A} 3537$ (Biogen*R) e diluição 1:500, anti-CD-34 humana (CD34- endothelial cell marker- Ab-1 clone QBEnd/10) monoclonal de rato na concentração $1.0 \mu \mathrm{g} / \mathrm{mL}$ cat.\# MS-363-R7 fabricado por LAB VISION Corporation, Westinghouse Dr. Fremont CA 94539 USA.

O equipamento computadorizado - sistema SAMBA 4000 que foi utilizado para na análise da imunomarcação, transforma as imagens analógicas em imagens numéricas. O software Immuno as interpreta e verifica duas variáveis preferenciais para caracterizar quantitativamente as expressões dos marcadores: índice de marcagem, densidade óptica média.

O índice de marcagem descreve o percentual de área tecidual da lâmina marcada pela reação antígeno-anticorpo. A densidade óptica média expressa a intensidade da reação antígeno-anticorpo.

As leituras foram realizadas com rastreamento dos campos visuais para localização dos "hot spots" e o procedimento de leitura em sentido de barra grega.

Os 26 pacientes com adenocarcinoma gástrico analisados, o foram por pelo menos um dos marcadores (caspase-3 $\mathrm{n}=22$ e CD-34 $\mathrm{n}=26$ ).

Para cada marcador foram avaliados o índice de marcagem (em percentual) e a densidade óptica. Os tumores foram classificados em relação à curvatura gástrica (grande ou pequena curvatura), diferenciação do tumor (pouco ou bem diferenciado), Bormann (0, I, II, III, IV), padrão de Lauren (Intestinal e difuso) e invasão linfática (NO ou N1/N2). Também foram consideradas as variáveis sexo e idade (menor ou igual a 60 e maior de 60) do paciente. Os tumores proximais e distais foram estudados avaliando-se a expressão, quantificação e comparação dos marcadores.

Os resultados foram expressos por médias, medianas, valores mínimos, valores máximos e desvios-padrão. Para estimar a média das expressões dos marcadores foram construídos intervalos de $95 \%$ de confiança. A avaliação da condição de normalidade das variáveis foi feita pelo teste de Shapiro-Wilks. Na comparação dos marcadores usou-se o teste $t$ de Student para amostras dependentes. A comparação entre dois grupos de pacientes foi feita pelo teste não paramétrico de Mann-Whitney. A comparação entre três grupos foi feita com o teste não-paramétrico de Kruskal-Wallis. $\mathrm{N}$ análise da correlação entre marcadores estimou-se o coeficiente de correlação de Pearson. Valores de $P<0,05$ indicaram significância.

\section{RESULTADOS}

Quanto ao sexo e idade, foram incluídos 16 homens e 10 mulheres com idades médias de 61 e 38 anos, respectivamente.

A expressão dos marcadores foram altas tanto para o índice de marcagem quanto para a densidade óptica com maior expressão da caspase-3. Especificamente, o índice de marcagem da caspase-3 $(\mathrm{n}=22)$ foi de $87.72 \% \pm 7.89$ enquanto a densidade óptica, $60.75 \pm 7.46$. A densidade óptica do CD34 ( $\mathrm{n}=26)$ foi de $84.46 \% \pm 9.17$, enquanto a densidade óptica, $49.84 \pm 8.48$.

A média da expressão da caspase- 3 foi similar a da média de CD34 e sem diferença significativa $(P=0.468)$.

Na comparação entre os marcadores em relação à densidade óptica (Figura 1) a média da caspase-3 foi maior do que a do CD34 e apresentou diferença fortemente significativa entre eles $(P<0.001)$.

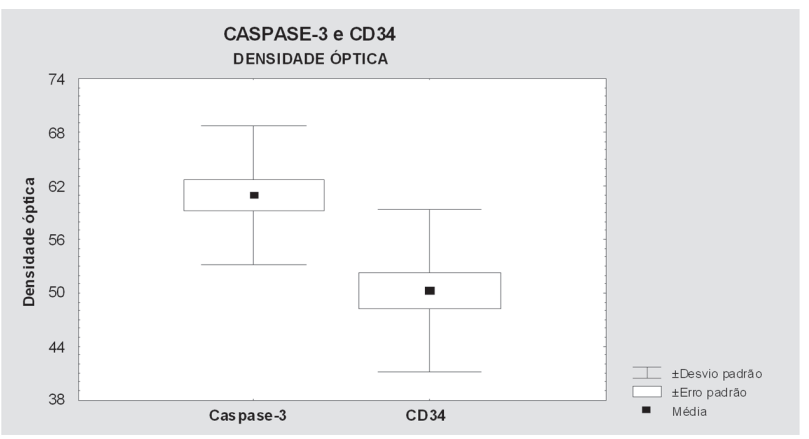

FIGURA 1 - Comparação entre os marcadores caspase-3 e CD34 em relação à densidade óptica

Na correlação entre o índice de marcagem dos marcadores (Figura 2), a análise da correlação foi linear negativa e não foi significativa com $\mathrm{r}=-0.262$ e $P=0.265$. 
Na correlação entre densidade óptica dos marcadores, a análise da correlação não foi significativa com $\mathrm{r}=0.234$ e $P=0.321$.

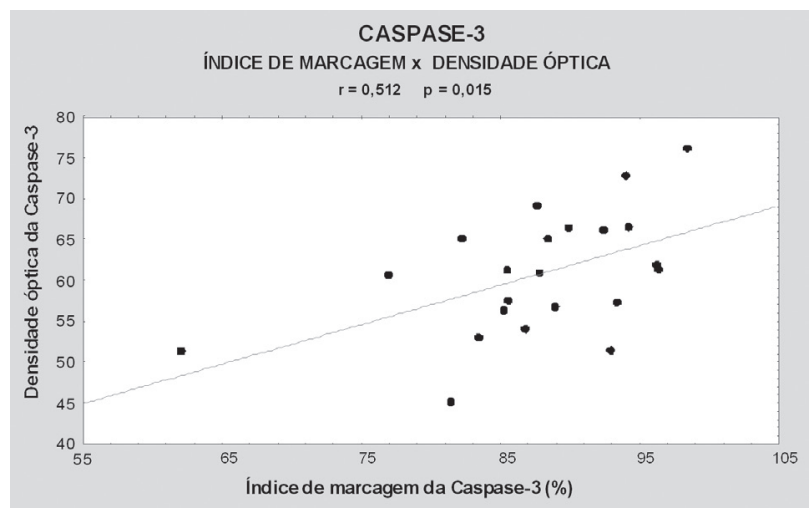

FIGURA 2 - Correlação da caspase-3 comparando a densidade óptica versus o índice de marcagem

Na correlação da caspase-3 comparando a densidade óptica versus o índice de marcagem (Figura 2) os resultados apresentaram diferenças significativas com $\mathrm{r}=0.512 \mathrm{e}$ $P<0.015$ e representa correlação linear positiva.

$\mathrm{Na}$ comparação do $\mathrm{CD} 34$ relativo à densidade óptica versus índice de marcagem os resultados apresentaram diferenças muito significativas com correlação linear positiva para $\mathrm{r}=0,512, P<0.015 \mathrm{e}$ $\mathrm{r}=0.520, P<0.006$, respectivamente. Chama a atenção nesta correlação, a correspondência do índice de marcagem de CD34 alto e densidade de CD34 alta, mas quando analisa-se a expressão dos marcadores vê-se que o índice de marcagem sempre é maior do que a densidade óptica.

Os resultados não mostraram diferenças significativas nos tumores da pequena e grande curvatura do estômago $(P>0.005)$. Observou-se que houve maior expressão da média da caspase-3, tanto para a marcagem quanto para a densidade, dos tumores da grande curvatura do estômago.

Os resultados não mostram diferença significativa nos tumores da pequena e da grande curvatura do estômago $(P>0.05)$. Notou-se que a média do índice de marcagem e a densidade óptica do CD34 era mais elevado para os tumores localizados na pequena curvatura do estômago.

Quanto à diferenciação tumoral, os resultados não apresentaram diferenças significativas $(P>0.05)$. Observou-se que a média do índice de marcagem e a densidade óptica de caspase-3 foi maior para os tumores bem diferenciados. Quanto ao CD34, os resultados não apresentam diferença significativa entre os tumores pouco e bem diferenciados, mas a densidade do CD34 foi também maior para os bem diferenciados.

Na classificação de Borrmann versus expressão dos marcadores, o comportamento da caspase- 3 expressou-se crescentemente quanto ao índice de marcagem com estádio de Borrmann 0 a IV. A comparação separada dos graus de Borrmann entre si não mostrou diferença significativa (Tabela 1).
TABELA 1 - Comparação da expressão do índice de marcagem e da densidade de CD34 nos tumores Borrmann divididos por categorias

\begin{tabular}{llllll}
\hline Marcador & $\mathbf{N}$ & Borrmann & $\mathbf{M} \pm \mathbf{D P}$ & & $\boldsymbol{P}^{*}$ \\
\hline ICD & 4 & 0 / I & $92,03 \%$ & 5,13 & 0,159 \\
& 5 & II & $81,80 \%$ & 7,38 & \\
& 16 & III / IV & $84,03 \%$ & 10,14 & \\
DCD & 4 & $0 /$ I & 53,50 & 7,13 & 0,496 \\
& 5 & II & 45,74 & 10,87 & \\
& 16 & III / IV & 50,44 & 8,22 & \\
\hline
\end{tabular}

*Teste não paramétrico de Kruskal-Wallis

* Teste não aplicado em função do pequeno número de casos.

Os resultados não mostram diferenças significativas entre os tumores de padrão difuso e intestinal $(P>0.05)$, mas os de padrão intestinal apresentaram índice de marcagem e densidade óptica da caspase-3 maiores do que os

TABELA 2 - Comparação da expressão do índice de marcagem da caspase-3 e a densidade óptica nos tumores tipos difuso e intestinal

\begin{tabular}{llllll}
\hline Marcador & $\mathbf{N}$ & Padrão & $\mathbf{M} \pm \mathbf{D P}$ & & $\boldsymbol{P}^{*}$ \\
\hline IC & 10 & Difuso & $85,67 \%$ & 9,66 & 0,173 \\
& 11 & Intestinal & $90,55 \%$ & 4,73 & \\
\multirow{2}{*}{ DC } & 10 & Difuso & 60,52 & 10,22 & 0,756 \\
& 11 & Intestinal & 60,96 & 4,76 & \\
\hline
\end{tabular}

*Teste não paramétrico de Kruskal-Wallis.

difusos (Tabela 2).

Os resultados não mostram diferenças significativas entre os tumores de padrão difuso e intestinal $(P>0.05)$ quanto ao índice de marcagem do CD34. Contudo, os

TABELA 2 - Comparação da expressão do índice de marcagem da caspase-3 e a densidade óptica nos tumores tipos difuso e intestinal

\begin{tabular}{llllll}
\hline Marcador & $\mathbf{N}$ & Padrão & $\mathbf{M} \pm \mathbf{D P}$ & & $\boldsymbol{P}^{*}$ \\
\hline ICD & 10 & Difuso & $84,49 \%$ & 10,34 & 0,893 \\
& 14 & Intestinal & $85,16 \%$ & 8,91 & \\
\multirow{2}{*}{ DCD } & 10 & Difuso & 50,88 & 6,26 & 0,979 \\
& 14 & Intestinal & 49,29 & 9,13 & \\
\hline
\end{tabular}

*Teste não paramétrico de Kruskal-Wallis.

tumores padrão intestinal foram maiores do que os difusos.

Quanto ao sexo, os resultados não mostraram diferenças significativas entre os parâmetros dos marcadores, mas demonstrou predomínio da expressão do índice de marcagem e densidade óptica para os pacientes do sexo feminino.

Observou-se que a média tanto do índice de marcagem quanto da densidade óptica para caspase-3 e CD43 foram maiores para os tumores sem invasão linfática quando comparados aos com invasão.

Os resultados mostraram diferença significativa entre os tumores proximais e distais onde a média da densidade do CD34 foi maior nos tumores distais do que nos tumores proximais. 
Quanto à idade não houve diferenças significativas entre tumores de pacientes com idade até 60 anos e com mais de $60(P>0.05)$. Contudo, observou-se que o índice de marcagem foi maior que a densidade óptica nos dois marcadores e que não houve correlação significativa entre a idade e a expressão dos marcadores $(P>0.05)$.

\section{DISCUSSÃO}

A captação de material para este estudo apresentou algumas dificuldades na seleção de material apropriado para análise devido à falta de protocolo criterioso no processo de parafinização dos tecidos na confecção dos blocos e sua conservação em função de ter sido obtido material de arquivo. Assim, em pesquisas que usam este processo metodológico é necessário que se adicione número de casos muito maior que o necessário, pois a exclusão é de grande monta.

Aqui as expressões de caspase-3 e de CD34 foram elevadas no foco primário do adenocarcinoma gástrico com índice de marcagem maiores do que a densidade óptica. Segundo Kondo et al. ${ }^{5}$ a expressão de CD34 tem pouca especificidade por marcar tecidos benignos e malignos.

A comparação dos marcadores CD34 e caspase-3 em relação ao índice de marcagem não mostrou diferenças significativas, mas quando comparou-se os marcadores em relação à densidade óptica, encontrou-se diferença fortemente significante mostrando densidade de caspase-3 maior do que a do CD34.

Esses resultados ensejam discussão por se esperar que nos tumores as caspases estivessem inibidas e que proteinas transmembranas tipo II, não iniciariam a morte programada das células e o mFas (Fas membrana) induzindo a apoptose celular, refletiria em baixa expressão nos tumores de qualquer tecido.

A expressão da caspase-3 elevada sugere a existência de escapes imunes como a mutação por superexpressão de $\mathrm{p} 53$ e dos inibidores das caspases Bcl-2 e FAP-1 por resistência à apoptose.

Observou-se que a marcagem de caspase-3 foi maior do que a sua densidade sugerindo que a quantificação da densidade pode estar relacionada com o acúmulo de caspase-3 não ativadas no citoplamáticas e no núcleo propiciando elevada reação da anti-caspase.

As expressões de CD34 foram altas mesmo tendo sido os tumores do tipo intestinal e muito diferenciado em maior número, onde os trabalhos da literatura mostram a expressão negativa de CD34. A explicação para tal acontecimento é que a expressão de CD34 não está dependente da capacidade do tumor produzir fatores como adesão/coesão das moléculas, de motilidade das células tumorais, receptores de fatores de crescimento necessários para metástase hematogênica e não para a angiogênese.

\section{Adensidadeóptica noSAMBAea densidademicrovascular.}

A média da densidade microvascular nos trabalhos de Tenderenda et al. ${ }^{8}$ (2001) foi de $43,15 \pm 19.8$ por campo de 200X e a densidade óptica neste estudo foi de $49,84 \pm 8.48$, valores muito próximos.

Sugere-se correlação entre a densidade microvascular e a densidade óptica marcada por CD34 encontrada neste estudo, onde a média dos números de vasos contados reflete o grau de angiogênese e a intensidade da reação do anticorpo anti-CD reflete a expressão da glicoproteína no reconhecimento dos pequenos vasos ou intensidade da angiogênese.

Assim como em outros trabalhos ${ }^{1,3,8}$ onde a marcação do CD34 não se correlacionou com a maioria dos fatores clínicopatológicos, exceto pela sobrevida e a ascite, neste estudo também não foi possível encontrar correlação do índice de marcagem e da densidade óptica com a curvatura gástrica, diferenciação do tumor, padrão tumoral, invasão linfática, Borrmann, sexo e idade dos pacientes.

\section{Expressão dos marcadores e a diferenciação do tumor}

Na expressão de caspase-3 e CD34 dos tumores pouco diferenciados e muito diferenciados, o índice de marcagem e a densidade óptica não mostraram diferenças significativas. Tenderenda et al. ${ }^{8}$ relataram íntima relação entre a vascularização tumoral e o grau do tumor. Estes autores observaram forte correlação positiva entre angiogênese e câncer gástrico, medida pela expressão de CD34 e o grau de diferenciação histológica com tumores pobremente diferenciados, tendo expressão de CD34 maior do que os bem e moderadamente diferenciados o que não foi observado neste estudo

\section{Expressão dos marcadores e o padrão do tumor}

Quando avaliou-se a expressão dos marcadores com o padrão do tumor, os resultados não mostraram diferenças significativas e são concordantes com os de Ding et al. ${ }^{1}$ onde CD34 não se correlacionou com os parâmetros clínico-patológicos exceto com sobrevida e ascite. Já os resultados de Nakayama et al. ${ }^{6}$ mostraram que nos tumores difusos e avançados, com invasão da muscular própria e a subserosa, o $\mathrm{CD} 34$ foi fortemente positivo. Nos tumores difusos e precoces o CD34 foi negativo.

Nos casos de tumores intestinais, independentes da profundidade do tumor, a expressão de CD34 foi negativa. Este comportamento sugere que a expressão de CD34 está associada com a progressão dos carcinomas tipo difuso e que a ausência desta expressão é também vista no tipo intestinal que estão progredindo.

Ellis² relatou que a angiogênese no câncer gástrico difuso é mais baixa do que no câncer gástrico intestinal (propagação tipicamente hepática e volumosa). A contagem dos vasos correlaciona-se com o estágio da doença e a formação de metástase em contraste a contagem de vasos no tipo difuso (propagação por implantes peritoniais e pequeno) não se correlaciona com metástase (não requer alto grau de angiogênese).

Esta controvérsia é explicada pelo uso de diferentes metodologias para contagem dos vasos. Yoo et al. ${ }^{9}$ relatou alta expressão de caspase- 3 no tipo difuso ( $92 \%$ de marcação) e no tipo intestinal ( $97 \%$ de marcação).

\section{Expressão dos marcadores em relação ao sexo e a invasão linfática}

Com estes marcadores não se encontrou correlação significativa entre o sexo e a expressão pelo índice de marcagem e a 
densidade óptica. Zheng et al. ${ }^{10}$ relataram que a expressão de caspase- 3 em células de metástase é maior do que em células de tecidos adjacentes ao tumor e no foco tumoral. Os resultados deste estudo não apresentam correlação da expressão dos marcadores com a invasão linfática. Tumores gástricos que apresentam altos índices de angiogênese e que ainda não expressaram outros fatores para formação de metástase - adesão/coesão das moléculas, fatores de motilidade, receptores de fatores de crescimento -, têm expressão elevada destes marcadores sem correlação com invasão linfática.

\section{Expressão e quantificação dos marcadores e o local do tumor e idade}

Para o local do tumor no estômago observa-se que a média da marcagem e da densidade óptica da caspase-3 foi maior do que a média do CD34. Os resultados deste estudo apresentaram diferença significativa entre os tumores com localização distal e proximal quando se compara a expressão da densidade óptica do CD34.

A correlação do índice de marcagem do CD34 com o da caspase-3 foi linear negativa, a correlação da densidade óptica do CD34 com a densidade óptica de caspase- 3 foi linear positiva, mas ambos não apresentaram resultados significantes. Já a correlação da densidade óptica com o índice de marcagem tanto de caspase-3 quanto de CD34 apresentaram resultados significantes com correlação linear positiva. Este fato sugere que os altos índices de marcagem para ambos os tumores expressa o nível de caspase-3 não ativada

Há relatos ${ }^{4,7}$, que a incidência dos cânceres gástricos distais (corpo e antro) tem diminuído no mundo ocidental enquanto o proximal (cárdia e gastroesofágico) tem aumentado mais do que qualquer outro câncer. Esses tumores são morfologicamente indistinguíveis e a metaplasia intestinal parece ter importante papel na carcinogênese de ambos tumores. As diferenças epidemiológicas observadas são na via genética da carcinogênese. No entanto são pobremente avaliadas.

Os resultados não mostraram diferenças significativas entre os parâmetros estudados em pacientes de até 60 anos e os com mais de 60 anos, nem correlação entre a idade e a expressão dos marcadores.

\section{CONCLUSÃO}

1. A densidade óptica média da caspase-3 é maior do que a do CD34. Há correlação linear positiva entre a densidade óptica média e o índice de marcagem tanto para CD34 quanto para caspase-3. 2.Não há correlação entre a expressão e quantificação dos marcadores e os fatores clínicos-patológicos avaliados (curvatura gástrica, diferenciação, Borrmann, padrão, invasão linfática, sexo e idade). 3. A densidade óptica de CD34 do adenocarcinoma gástrico distal é maior do que a dos proximais, sugerindo que apresentam diferenças biológicas relacionada com a localização do tumor.

Silva PHFF, Cuenca RM, Ribas-Filho JM, Ribas CAPM, Malafaia O, Dobrowolski S, Bora FR. CD34 and caspase-3: expression, quantification comparison and correlation in gastric adenocarcinoma. ABCD Arq Bras Cir Dig 2008;21(4): 180-4

ABSTRACT - Background - Gastric cancer continues to defy the modern medicine in the understanding of its biological behavior and surgical cure. Aim - To determine, compare and correlate the expression of caspase-3 and CD34 in gastric adenocarcinoma with clinical and pathological factors and proximal/distal gastric tumors. Methods - Imunohistochemistry quantitative analysis of the antibodies anti-caspase-3 polyclonal of rabbit and CD43 mouse's monoclonal, to mark adenocarcinoma cells in 26 CD34's cases and 22 caspase-3 paraffined blocks were used. The parameters were: labeling index in percentage and optic density. Results - Caspase-3 and CD34 expression were elevated; the average and the deviation standard for labeling index were respectively $87,72 \% \pm 7,89$ and $84,86 \% \pm 9,17$; for optic desity $60,75 \pm 7,46$ and $49,84 \pm 8.48$. When markers were compared with optic density it was found significant differences between caspase-3 $(60,94)$ and CD34 $(50,27)$. Caspase-3 average $(60,94)$ was higher than CD34 $(50,27), P<0.001$. The correlation among markers was positive for CD34's optic density versus labeling index of CD34 $(P=0.006, \mathrm{r}=0,520)$ and for optic density of caspase-3 versus caspase-3 labeling index $(P=0.015, \mathrm{r}=0.512)$. The markers had no correlation in the comparison of labeling index and optic densities with the gastric curvature, tumor differentiation, Borrmann classification and lymphatic invasion. Gender and age also did not correlate with the markers. The correlation with the tumor location had differences favoring the ones on distal part. Conclusion - Caspase-3 and CD34 expressions are elevated in gastric adenocarcinoma. Caspase-3 optic density is higher than CD34's optic density. There is no expression and quantification correlation between markers and the clinical and pathological factors, but there are evidences that they have biological differences when in different gastric anatomical parts.

HEADINGS: CD34. Caspase-3. Cytophotometry.

\section{REFERÊNCIAS}

1. Ding S, Li C, Lin S, Yang Y, Liu D, Han Y, Zhang Y, Li L, Zhou L, Kumar S. Comparative evaluation of microvessel density determined by CD34 or CD105 in benign and malignant gastric lesions. Hum Pathol. 2006 Jul;37(7):861-6.

2. Ellis LM, Fidler IJ. Angiogenesis and metastasis. Eur J Cancer. 1996 Dec;32A(14):2451-60.

3. Estrov Z, Thall PF, Talpaz M, Estey EH, Kantarjian HM, Andreeff M, Harris D, Van Q, Walterscheid M, Kornblau SM. Caspase 2 and caspase 3 protein levels as predictors of survival in acute myelogenous leukemia. Blood. 1998 Nov 1;92(9):3090-7.

4. Gulmann C, Grace A, Leader M, Butler D, Patchett S, Kay E. Adenomatous polyposis coli gene, beta-catenin, and E-cadherin expression in proximal and distal gastric cancers and precursor lesions: an immunohistochemical study using tissue microarrays. Appl Immunohistochem Mol Morphol. 2003 Sep;11(3):230-7.

5. Kondo K, Muramatsu M, Okamoto Y, Jin D, Takai S, Tanigawa N, Miyazaki $\mathrm{M}$. Expression of chymase-positive cells in gastric cancer and its correlation with the angiogenesis. J Surg Oncol. 2006 Jan 1;93(1):36-42.

6. Nakayama H, Enzan H, Miyazaki E, Kuroda N, Naruse K, Kiyoku H, Toi M, Hiroi M. CD34 positive stromal cells in gastric adenocarcinomas. J Clin Pathol. 2001 Nov;54(11):846-8.
7. Pacelli F, Papa V, Caprino P, Sgadari A, Bossola M, Doglietto GB. Proximal compared with distal gastric cancer: multivariate analysis of prognostic factors. Am Surg. 2001 Jul;67(7):697-703.

8. Tenderenda M, Rutkowski P, Jesionek-Kupnicka D, Kubiak R. Expression of CD34 in gastric cancer and its correlation with histology, stage, proliferation activity, p53 expression and apoptotic index. Pathol Oncol Res. 2001;7(2):129-34.

9. Yoo NJ, Kim HS, Kim SY, Park WS, Kim SH, Lee JY, Lee SH. Stomach cancer highly expresses both initiator and effector caspases; an immunohistochemical study. APMIS. 2002 Nov;110(11):825-32.

10. Zheng HC, Sun JM, Wei ZL, Yang XF, Zhang YC, Xin Y. Expression of Fas ligand and caspase- 3 contributes to formation of immune escape in gastric cancer. World J Gastroenterol. 2003 Jul;9(7):1415-20.

\footnotetext{
Fonte de financiamento: não há Conflito de interesse: não há Recebido para publicação: 30/052008 Aceito para publicação: 31/09/2008
} 